\title{
Review of the New American Cancer Society Guidelines for Breast Cancer Screening for Women at Average Risk
}

\author{
SYLVIA S. ESTRADA, DNP, MSN, MSHCM, WHNP-BC, CBCN
}

From Cedars-Sinai Medical Center, Samuel Oschin Comprehensive Cancer InstituteBrandman Breast Center, Los Angeles, California

Author's disclosures of potential conflicts of interest are found at the end of this article.

Correspondence to: Sylvia S. Estrada, DNP, MSN, MSHCM, WHNP-BC, CBCN, Cedars-Sinai Medical Center, 310 N. San Vicente Blvd., Room 318,

Los Angeles, CA 90048.

E-mail:Sylvia.Estrada@cshs.org

doi: 10.6004/jadpro.2016.7.5.8

(c) 2016 Harborside Press ${ }^{\oplus}$
J Adv Pract Oncol 2016;7:563-566

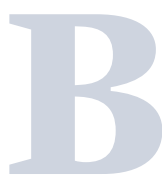

reast cancer is the most common cancer in women worldwide (Torre et al., 2015). In the United States, an estimated 231,840 women were diagnosed with breast cancer in 2015 (Siegel, Miller, \& Jemal, 2015). After lung cancer, breast cancer continues to rank second as a cause of cancer death in women in the United States as well as remains the leading cause of premature mortality for women. Even though death from breast cancer has declined steadily since 1990 , largely due to improvements in early detection and treatment (Berry et al., 2005), an estimated 40,290 women in the United States died of breast cancer in 2015 (Siegel et al., 2015).

In 2003, the American Cancer Society (ACS) issued its prior recommendations for breast cancer screening. The recommendations included annual mammography screening for all women starting at age 40 years and continuing as long as a woman remains in good health. Clinical breast examinations (CBEs) should be periodically performed for women in their 20s and 30 s and annually for women 40 years and older (Smith et al., 2003).
Since the 2003 ACS recommendations, new evidence has accumulated from long-term follow-up of randomized controlled trials and observational studies of organized, population-based screening programs. There is also greater emphasis on estimating the harms associated with screening; assessing the balance of benefits and harms; and supporting the interplay among values, preferences, informed decision-making, and recommendations (Oeffinger et al., 2015). The ACS has incorporated standards recommended by the Institute of Medicine into its guidelines development protocol to ensure a more trustworthy, transparent, and consistent process for developing and communicating guidelines (Brawley et al., 2011).

\section{DEFINING AVERAGE RISK}

The new guideline addresses recommendations for women at average risk. Average risk is defined as those women without a personal history of breast cancer, a confirmed or suspected genetic mutation known to be associated with increased risk 


\section{Table. Questions Guiding the Evidence Review of the ACS Breast Cancer Screening Guideline Update}

\section{Key questions}

Q1: What are the relative benefits, limitations, and harms associated with mammography screening compared with no screening among average-risk women 40 years and older, and how do they vary by age, screening interval, and screening history?

Q2: Among average-risk women who are screened with mammography, what are the relative benefits, limitations, and harms associated with annual, biennial, triennial, or another screening interval, and how do they vary by age?

Q3: What are the benefits, limitations, and harms associated with clinical breast examination (CBE) among average-risk women 20 years and older compared with no CBE, and how do they vary by age, interval, and participation rates in mammography screening?

\section{Other questions}

Q4a: Among women with an increased risk of breast cancer due to factors known prior to the onset of screening (e.g., family history, BRCA mutation carrier, history of chest irradiation), what are the relative benefits, limitations, and harms associated with different screening modalities compared with no screening (i.e., what ages to start and stop screening) and each other?

Q4b: Among women with an increased risk of breast cancer due to factors identified as the result of screening or diagnosis (e.g., prior diagnosis of proliferative lesions), what are the benefits, limitations, and harms associated with different screening modalities compared with no screening and each other?

Q5a: Among women with an increased risk of breast cancer due to factors known prior to the onset of screening (e.g., family history, BRCA mutation carrier, history of chest irradiation), what are the relative benefits, limitations, and harms associated with different screening modalities at different intervals, and how do they vary by age?

Q5b: Among women with an increased risk of breast cancer due to factors identified as the result of screening or diagnosis (e.g., prior diagnosis of proliferative lesions), what are the benefits, limitations, and harms associated with different screening modalities at different intervals, and how do they vary by age?

Note. Information from Oeffinger et al. (2015).

(e.g., $B R C A$ ), or a history of radiotherapy to the chest at a young age (Oeffinger et al., 2015). These investigators acknowledged there are also women outside these higher-risk categories who are at higher-than-average risk of breast cancer. Mammography screening alone may have reduced ef- fectiveness in these women. These higher-than-average risk women are those with significant family histories but without a high probability of carrying identified risk mutations, those with a prior diagnosis of benign proliferative breast, and those with significant mammographic breast density.

Intermediate-risk women may benefit from additional breast imaging other than screening mammography. At this time, the investigators have noted there are no reliable estimates of the number of women who have one or more of these risk factors; nor are there widely accepted riskbased screening recommendations that differ for women in this intermediate-risk group compared with average-risk women.

\section{REVIEW OF THE LITERATURE: EVIDENCE SYNTHESIS}

The ACS organized an interdisciplinary guidelines development group (GDG) consisting of clinicians, biostatisticians, epidemiologists, an economist, and patient representatives to develop the new ACS guidelines for women at average risk (Oeffinger et al., 2015). The ACS GDG selected the Duke University Evidence Synthesis Group to conduct the independent systematic evidence review of the breast cancer screening literature. Formulation of recommendations was based on the quality of the evidence and judgment (incorporating values and preferences) about the balance of benefits and harms.

The evidence-based breast cancer screening guideline for women at average risk focused on three key questions out of the five key questions (see Table). Key points from the evidence synthesis follow:

- Screening mammography in women aged 40 to 69 years is associated with reduction in breast cancer deaths across a range of study designs; inferential evidence supports breast cancer screening for women aged $\geq 70$ years who are in good health.

- Estimates of the cumulative lifetime risk of false-positive examination results are greater if screening begins at a younger age, due to the greater number of mammograms as well as to the higher recall rate for younger women. The quality of evidence for overdiagnosis is not sufficient to estimate a lifetime risk with confidence. 
- Analysis of the effects of the screening interval indicates more favorable tumor characteristics when premenopausal women are screened annually vs. biennially.

- Evidence does not support routine clinical breast examination as a screening method for women at average risk.

\section{UPDATED RECOMMENDATIONS}

The updated recommendations are based on the GDG's consensus judgment about when the benefits of mammography screening clearly or likely outweigh the harms in a population of women at average risk. These recommendations are designated as either strong or qualified. A strong recommendation indicates consensus that the benefits of adherence to an intervention outweigh the undesirable effects that may result from screening. A qualified recommendation indicates clear evidence of benefit of screening but less certainty about the balance of benefits and harms or patient values and preferences. These factors could lead to different decisions about screening (Oeffinger et al., 2015).

\section{Recommendation 1}

Women with an average risk of breast cancer should undergo regular screening mammography starting at age 45 years (Strong Recommendation).

1a. Women aged 45 to 54 years should be screened annually (Qualified Recommendation).

$1 b$. Women 55 years and older should transition to biennial screening or have the opportunity to continue screening annually (Qualified Recommendation).

1c. Women should have the opportunity to begin annual screening between the ages of 40 and 44 years (Qualified Recommendation).

\section{Recommendation 2}

Women should continue screening mammography as long as their overall health is good and they have a life expectancy of 10 years or longer (Qualified Recommendation).

\section{Recommendation 3}

The American Cancer Society does not recommend clinical breast exam for breast cancer screening among average-risk women at any age (Qualified Recommendation).

\section{Limitations}

The investigators acknowledged there were inevitable gaps between the available evidence and the evidence needed for the development of guidelines that precisely quantify and weigh the benefits vs. the harms associated with breast cancer screening. These gaps need further research to help women make screening decisions. Better evidence about the extent of overdiagnosis is important, as is more information about the preferences and decision processes of diverse populations (Keating \& Pace, 2015).

\section{CONCLUSION}

The new ACS recommendations are made in the context of maximizing reduction in breast cancer mortality and reducing years of life lost while minimizing the associated harms among the population of women in the United States. The ACS affirms that screening mammography saves lives. Fewer women will die of breast cancer as a result of early detection from routine screening mammography. The ACS also recognizes that the balance of benefits and harms will be close in some instances and that the spectrum of women's values and preferences will lead to varying decisions. The intention of this new guideline is to provide both guidance and flexibility for women about when to start and stop screening mammography and how frequently to be screened for breast cancer (Keating \& Pace, 2015).

Advanced practitioners need to understand the content and message in the new ACS breast cancer screening guidelines. When counseling averagerisk women older than 40 years, it is important to remember and emphasize there is no single right answer to the question "Should I have a mammogram?" Instead, women should be supported in estimating and understanding their risk of developing breast cancer and exploring their values and preferences so health-care providers can help them make informed decisions.

\section{Disclosure}

The author has no potential conflicts of interest to disclose. 


\section{References}

Berry, D. A., Cronin, K. A., Plevritis, S. K., Fryback, D. G., Clarke, L., Zelen, M.,...Feurer, E. J. (2005). Effect of screening and adjuvant therapy on mortality from breast cancer. New England Journal of Medicine, 353(17), 17841792. http://dx.doi.org/10.1056/NEJMoa050518

Brawley, O., Byers, T., Chen, A., Pignone, M., Ransohoff, D., Schenk, M.,...Wender, R. (2011). New American Cancer Society process for creating trustworthy cancer screening guidelines. Journal of the American Medical Association, 306(22), 2495-2499. http://dx.doi.org/10.1001/ jama.2011.1800

Keating, N. L., \& Pace, L. E. (2015). New guidelines for breast cancer screening in US women. Journal of the American Medical Association, 314(15), 1569-1571. http://dx.doi. org/10.1001/jama.2015.13086
Oeffinger, K. C., Fontham, E. T. H., Etzioni, R., Herzig, A., Michaelson, J. S., Shih, Y. C. T.,...Wender, R. (2015). Breast cancer screening for women at average risk 2015 guideline update from the American Cancer Society. Journal of the American Medical Association, 314(15), 1599-1614. http://dx.doi.org/10.1001/jama.2015.12783

Siegel, R. L., Miller, K. D., \& Jemal, A. (2015). Cancer statistics, 2015. CA: A Cancer Journal for Clinicians, 65(1), 5-29. http://dx.doi.org/10.3322/caac.21254

Smith, R. A., Saslow, D., Sawyer, K. A., Burke, W., Costanza, M. E., Evans, W. P.,...Sener, S. (2003). American Cancer Society guidelines for breast cancer screening: Update 2003. CA: A Cancer Journal for Clinicians, 53(3), 141-169. http:// dx.doi.org/10.3322/canjclin.53.3.141

Torre, L. A., Bray, F., Siegel, R. L., Ferlay, J., Lortet-Tieulent, J., \& Jemal, A. (2015). Global cancer statistics, 2012. CA: A Cancer Journal for Clinicians, 65(2), 87-108. http://dx.doi. org/10.3322/caac.21262

See page 567 for the related article by Karen Meneses 


\title{
When Should I Have a Mammogram? Recent Changes in ACS Mammography Guidelines: Implications for Practice
}

\author{
KAREN MENESES, PhD, RN, FAAN
}

From The University of Alabama at Birmingham

Author's disclosures of potential conflicts of interest are found at the end of this article.

Correspondence to: Karen Meneses, PhD, RN, FAAN, Professor and Associate Dean for Research, School of Nursing, The University of Alabama at Birmingham, 1720 Second Avenue South, Birmingham, AL 35294.

E-mail: menesesk@uab.edu

doi: 10.6004/jadpro.2016.7.5.9

(c) 2016 Harborside Press ${ }^{\oplus}$ $\mathrm{n}$ the United States, breast cancer is the most common female cancer and the second leading cause of female cancer death (American Cancer Society [ACS], 2015). Breast cancer significantly contributes to morbidity and premature mortality among American women (ACS, 2015). Early detection with mammography has been known to reduce morbidity and mortality. Yet, today, the answer to the routine question asked by millions of American women: "When should I have a mammogram?" requires a more complex answer. And the answer is "That depends."

\section{UPDATES}

In 2015, the ACS issued an update to its 2003 screening mammography guidelines (Oeffinger et al., 2015). The 2003 mammography screening recommendation for women at average risk was to start annual mammography at age 40 years and continue annual screening as long as a woman was in good health and, if diagnosed, could tolerate breast cancer treatment. The 2003 ACS guidelines also recommended that for women in their 20s and 30s, clinical breast examination (CBE) should be part of health evaluation at least every 3 years and for asymptomatic women 40 years and older, CBE should be performed annually.

The newly issued 2015 ACS screening mammography guidelines depart from the 2003 guidelines in several ways (Smith et al., 2003). First, annual screening recommendations differ based on age. The ACS now recommends that annual screening should begin at age 45 and continue until age 54 . At age 55 , women should transition to biennial screening and can continue as long as their overall health is good and they have a life expectancy of 10 or more years. Clinical breast examination is no longer recommended for every woman. Table 1 compares the ACS 2003 and 2015 guidelines.

So what prompted this substantial change in recommended screening guidelines? An editorial by Keating and Pace (2015) identified three key areas. First, the ACS commissioned a panel to review 
the current evidence of the benefits of mammography. The review panel included recent observational studies showing a wider range of estimates-about $20 \%$ to $40 \%$-for breast cancer mortality relative risk reductions compared with prior reviews of the evidence using randomized clinical trials showing risk reduction of about $15 \%$. The panel further acknowledged the limitations in both the observational designs (i.e., noncomparable control groups, lead time, and length of time bias) and the randomized trial designs (i.e., heterogeneous, older, and increasingly outdated).

Second, the panel used the Surveillance, Epidemiology, and End Results (SEER) data using 5-year age groups to estimate the benefits and harms of mammography screening compared with previous estimates using 10-year age groups. The panel found that the breast cancer deaths between women aged 45 to 49 years compared with those of women aged 50 to 54 years, rather than women aged 40 to 44 years. Thus, the panel recommended screening mammography to begin at age 50 .

Third, the panel commissioned an analysis of annual vs. biennial screening by the Breast Cancer Surveillance Consortium (BCSC; Miglioretti et al., 2015). The BCSC evaluated 15,440 women aged 40 to 85 diagnosed with breast cancer between 1996 and 2012. Women received either annual or biennial screening mammography. Findings showed that premenopausal women with biennial screening had higher stage IIIB disease com- pared with women having annual screening. No differences were found between premenopausal and postmenopausal women. Thus, the panel recommended the change in the ACS guideline that women between 45 and 54 years should receive annual screening.

The panel found that the evidence of $\mathrm{CBE}$ and mortality reduction with CBE to be of low quality and unconvincing. Thus, the panel recommended against $\mathrm{CBE}$.

\section{AVERAGE vs. HIGH RISK}

Overall, the changes in the ACS recommendations refer to women of average breast cancer risk. So, who are they? Average-risk women are those who have none of the following characteristics: (1) a personal history of breast cancer; (2) a suspected or confirmed genetic mutation such as $B R C A$; and (3) a personal history of radiation therapy to the chest wall received at a young age. The recommendations do not refer to women who are considered at higher risk.

Who is considered higher risk among women younger than 45 years? They are women with (1) a personal history of breast cancer; (2) a suspected or confirmed genetic mutation such as $B R C A$; (3) personal history of radiation therapy to the chest wall received at a young age; (4) first-degree relatives diagnosed with breast cancer before the age of 45 or ovarian cancer at any age (particularly if more than one relative was diagnosed) or a male relative with breast cancer; (5) Ashkenazi Jewish heritage; and/ or (6) other breast health problems or conditions,

\section{Table 1. Comparison of 2003 and 2015 ACS Breast Cancer Screening Guidelines for Average-Risk Women 2003 Guideline 2015 Guideline \\ Annual screening Begin by age 40 and continue through life as long as a woman is in reasonably good health \\ Biennial screening \\ Clinical breast exam \\ Breast self-examination \\ At least every 3 years for women aged 20-30; annually for asymptomatic women 40 years and older \\ No statement \\ Have the opportunity to start annual screening at age 40-44 years; start annual screening at age $45-54$ years \\ Start at age 55 or have the opportunity to continue as long as overall health is good and life expectancy is 10 years or longer \\ Not recommended \\ No statement}

Note. ACS = American Cancer Society. 
such as breast density, lobular carcinoma in situ, ductal carcinoma in situ, atypical ductal hyperplasia, or atypical lobular hyperplasia.

\section{COMPARING SCREENING GUIDELINES}

How do the 2015 ACS mammography screening guidelines compare with the 2009 US Preventive Services Task Force (USPSTF) recommendations? Table 2 compares the two guidelines related to annual and biennial screening, $\mathrm{CBE}$, and breast self-exam. First, the USPSTF recommends biennial screening mammography for women between the ages of 50 and 74. Deciding to start regular, biennial screening mammography before the age of 50 should be done individually taking context into account, including patients' values about benefits and harms.

Second, the USPSTF does not recommend screening mammography in women 75 and older. Third, the USPSTF found insufficient evidence to recommend CBE in women over 40. Fourth, the USPSTF recommends against teaching breast selfexam. And finally, current evidence regarding digital mammography or magnetic resonance imaging is insufficient to assess the additional benefits and harms over film mammography.

\section{IMPLICATIONS FOR ADVANCED PRACTITIONERS}

So we return to the question, "When should I have a mammogram?" The answer can still be "that depends," because there is no specific right or wrong answer for women of average risk. Although some advanced practitioners may argue that the recommendations are confusing, it is hoped that others may view the new 2015 ACS recommendations in another light: one where the dialogue becomes patient-driven, patientcentered, and patient-valued.

Advanced practitioners can begin the conversation with patient-driven comments: "Let's talk about average risk. Are you at average risk? That depends on your current age as well as your family and personal histories. Maybe you are not at average risk. Thus, the guidelines do not pertain to you." It may be likely that advanced practitioners in oncology may see women who are not at average risk. They may be seeing survivors and family members of cancer survivors.

Among our advanced practitioner colleagues in primary care or women's health practices, they may be using the USPSTF guidelines for averagerisk women. In this instance, the USPSTF guidelines may be more in line with the updated ACS guidelines, where average-risk women can have the opportunity to start biennial screening before the age of 50 years. The USPSTF guidelines are silent on the topic of annual screening before the age of 50, but the 2015 ACS guidelines for average-risk women begin at age 45 . Thus, it is most likely that the topic of screening average-risk young women is covered in the ACS guidelines. So, both the new ACS and the USPSTF guidelines for young women cover the answer to "When should I have a mammogram?" Most importantly, maintaining a balanced discussion focused on risk and benefits as well as personal preferences and values can keep the dialogue moving.

Table 2. Comparison of 2015 ACS and 2009 USPSTF Breast Cancer Screening Guidelines

\section{USPSTF Guideline}

Annual screening

Biennial screening

Breast self-examination
Have the opportunity to start screening before age 50 depending on contextual factors; start screening at age 50 and continue to age 74 years; end screening at age 75 years

Screening not recommended after age 40

Do not teach BSE

\section{ACS Guideline}

Have the opportunity to start annual screening at age 40-44 years; start annual screening at age $45-54$ years

Start at age 55 or have the opportunity to continue as long as overall health is good and life expectancy is 10 years or longer

Not recommended

No statement

Note. ACS = American Cancer Society; USPSTF = US Preventive Services Task Force; BSE = breast self-exam. 
Given the weight of the new evidence about average risk, there is greater opportunity to apply evidence to practice to help women make informed decisions that are best for them. So, let the conversation begin!

\section{Disclosure}

The author has no potential conflicts of interest to disclose.

\section{References}

American Cancer Society. (2015). Cancer facts and figures 2015. Retrieved from http://www.cancer.org/acs/ groups/content/@editorial/documents/document/acspc-044552.pdf

Keating, N. L., \& Pace, L. E. (2015). New guidelines for breast cancer screening in US women. Journal of the American Medical Association, 314(15), 1569-1571. http://dx.doi. org/10.1001/jama.2015.13086
Miglioretti, D. L., Zhu, W., Kerlikowske, K., Sprague, B. L., Onega, T., Buist,...Smith, R. A. (2015). Breast tumor prognostic characteristics and biennial versus annual mammography, age, and menopausal status. Journal of the American Medical Association Oncology, 1(8), 1069-1077. http://dx.doi.org/10.1001/jamaoncol.2015.3084

Oeffinger, K. C., Fontham, E. T., Etzioni, R., Herzig, A., Michaelson, J. S., Shih, Y. C.,...Wender, R. (2015). Breast cancer screening for women at average risk: 2015 Guideline update from the American Cancer Society. Journal of the American Medical Association, 314(15), 1599-1614. http:// dx.doi.org/10.1001/jama.2015.12783

Smith, R. A., Saslow, D., Sawyer, K. A., Burke, W., Costanza, M. E., Evans, W. P. 3rd,...Sener, S. (2003). American Cancer Society guidelines for breast cancer screening: Update 2003. CA: A Cancer Journal for Clinicians, 53(3), 141-169. http://dx.doi.org/10.3322/canjclin.53.3.141

US Preventive Services Task Force. (2009). Screening for breast cancer: U.S. Preventive Services Task Force Recommendation Statement. Annals of Internal Medicine, 151(10), 716-726. http://dx.doi.org/10.7326/0003-4819151-10-200911170-00008 